\title{
MAGNETIC FLUX CONCENTRATION BY SIPHON FLOWS IN ISOLATED MAGNETIC FLUX TUBES
}

\author{
John H. Thomas \\ Department of Mechanical Engineering, Department of Physics and \\ Astronomy, and C. E. K. Mees Observatory \\ University of Rochester \\ Rochester, New York 14627, U.S.A.

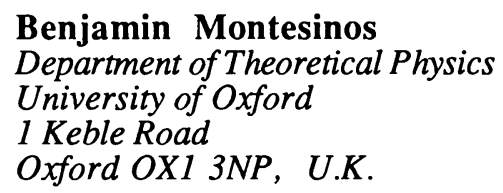

ABSTRACT. Siphon flows along arched, isolated magnetic flux tubes, connecting photospheric footpoints of opposite magnetic polarity, cause a significant increase in the magnetic field strength of the flux tube due to the decreased internal gas pressure associated with the flow (the Bernoulli effect). These siphon flows offer a possible mechanism for producing intense, inclined, small-scale magnetic structures in the solar photosphere.

In order to produce magnetic flux tubes in the solar photosphere with the observed field strengths of $1000-1500 \mathrm{G}$, the interior of the flux tubes must be substantially evacuated. The observed magnetic field strengths are very near the limiting maximum value $B_{p}=\left(8 \pi p_{e}\right)^{1 / 2}$ of a totally evacuated flux tube confined by the external gas pressure $p_{e}$; thus, a mechanism that produces a substantial evacuation of the flux tube is required. One such mechanism, first proposed by Parker (1978), is the convective collapse of a vertical flux tube in the superadiabatic layer just below the photosphere. This mechanism has been studied extensively (see, for example, Webb and Roberts 1978; Spruit and Zweibel 1979; Spruit 1979; Hasan 1985; Venkatakrishnan 1985; Nordlund 1986; and the recent reviews by Solanki 1987, Stenflo 1989, and Thomas 1989). Here we discuss another possible mechanism, namely, siphon flows in an arched, isolated magnetic flux tube (Thomas 1984, 1988).

Earlier work on siphon flows, beginning with Meyer and Schmidt (1968), dealt exclusively with the case of an effectively rigid, embedded flux tube in the limit of low plasma beta, appropriate for conditions in the solar corona or chromosphere (see the review by Priest 1981). For conditions in the solar photosphere or convection zone, with plasma beta of order unity, the flux tube cannot be considered rigid; its cross-sectional area and magnetic field strength must change in response to changes in internal gas pressure induced by the siphon flow in order to maintain lateral pressure balance with the surrounding atmosphere. The reduced internal gas pressure associated with the siphon flow (the Bernoulli effect) results in an increased magnetic field strength and a reduced crosssectional area of the flux tube.

The critical speed for siphon flows in a thin isolated flux tube is the so-called tube speed $c_{t}=\left[c^{2} a^{2} /\left(c^{2}+a^{2}\right)\right]^{1 / 2}$, where $c$ is the internal sound speed and $a$ is the Alfvén speed. 
(The tube speed is the speed of propagation of a longitudinal, or sausage-mode, wave along a thin isolated magnetic flux tube; it is smaller than either the sound speed or the Alfvén speed.) Flows with speeds less than $c_{t}$ (subcritical flows) or greater than $c_{t}$ (supercritical flows) along an isolated flux tube are qualitatively equivalent to subsonic or supersonic flows along a rigid, embedded flux tube. In an arched flux tube, the flow can undergo a smooth transition from subcritical to supercritical speed only at the top of the arch where the tube is locally horizontal.

The maximum speed of the siphon flow depends on the height of the arch and the initial velocity at the upstream footpoint. If the arch height is small enough or the initial velocity is low enough, the flow remains subcritical throughout. If the maximum velocity is less than the characteristic speed $c_{1}=\left[c^{2} a^{2} /\left(2 c^{2}+a^{2}\right)\right]^{1 / 2}<c_{t}$, then the flow accelerates and the tube expands with increasing height all along the upstream leg of the arch, and the flow decelerates and the tube contracts with decreasing height all along the downstream leg of the arch. The expansion of the tube with height is less than it would be without the flow, however, due to the decreased internal pressure caused by the flow (the Bernoulli effect). If the maximum velocity exceeds the characteristic speed $c_{l}$ but is still less than $c_{t}$, the flow accelerates and the tube expands up to the point where $\mathrm{v}=c_{1}$ and beyond that point the flow accelerates but the tube contracts with increasing height in the upstream leg of the arch. This produces a point of local maximum cross-sectional area, or "bulge point," in the tube at the point where $\mathrm{v}=c_{1}$. In the downstream leg of the arch the flow decelerates symmetrically and there is another bulge point . For such a flow the Bernoulli effect is so strong that it more than compensates for the decreasing pressure with height outside the tube and causes the tube to contract with height above the bulge point.

If the arch height is increased or the initial velocity at the upstream footpoint is increased, we reach a critical flow in which $v=c_{t}$ at the top of the arch. In this case the flow can accelerate smoothly to supercritical velocity in the downstream leg of the arch. The flow velocity continues to increases while the cross-sectional area decreases (and the magnetic field strength increases) with decreasing height along the downstream leg of the arch. However, this supercritical flow requires a very small internal gas pressure at the downstream footpoint which will not be the case in the solar photosphere. For a higher value of the "backpressure" at the downstream footpoint, the flow will decelerate abruptly and the cross-sectional area will increase abruptly across a standing "tube shock" at some point along the downstream leg of the arch. The critical flow is "choked," in the sense that a decrease in the pressure at the downstream footpoint will not increase the mass flow rate along the tube; disturbances cannot propagate upstream along the supercritical section of the flow.

We have carried out extensive numerical computations of siphon flows in arched, isolated flux tubes in the limits of isothermal flow (Thomas 1988) and adiabatic flow (Monetsinos and Thomas 1989). Siphon flows are capable of increasing the magnetic field strength of an isolated flux tube up to as much as 80 or $90 \%$ of the limiting vacuum value and thus can, in principle, produce flux tubes with strengths $1500 \mathrm{G}$ or more in the solar photosphere. In general, adiabatic flows produce greater magnetic flux concentration than isothermal flows (Montesinos and Thomas 1989). Radiative transfer in a real photospheric flux tube will produce conditions somewhere in between isothermal and adiabatic flow.

The large-scale equilibrium shape of a flux tube arch containing a steady siphon flow is determined by a balance among the buoyancy force, the net magnetic tension force, and the inertial force associated with the siphon flow along curved streamlines (the centrifugal force). We have calculated the equilibrium shape of the flux tube arch for a wide variety of siphon flows (Thomas and Montesinos 1989) and compared it with the shape of a static arched flux tube first determined by Parker (1975). In general, the presence of a siphon flow requires that the arch be more highly curved in order that the net magnetic tension 
force can balance the additional effect of the centrifugal force. With increasing flow speed, the footpoints of the arch move closer together relative to the height of the arch. Provided there is some mechanism for anchoring the ends of the flux-tube arch somewhere in the convection zone, the intensified part of the flux tube at the top of the arch might be held in mechanical equilibrium in the photosphere for a time much longer than the rise time associated with the magnetic buoyancy alone.

A totally isolated flux tube arch is highly curved and the distance between the footpoints in the photosphere is at most equal to about six times the density scale height of the surrounding atmosphere. This configuration would produce closely spaced intense magnetic elements of opposite polarity in the photosphere. However, more widely spaced footpoints are possible if the arch reaches up to the overlying magnetic canopy. In this case, the upper part of the arch can extend horizontally over a considerable distance, being held down against its buoyancy force by the magnetic pressure of the canopy.

We suggest that siphon flows in arched magnetic flux tubes may be the cause of some of the intense magnetic fields observed in the solar photosphere outside sunspots. In these cases the observed magnetic field will be inclined to the vertical, with the inclination depending on the height of formation of the spectral line. Observations do indicate that a significant fraction of the photospheric flux tubes are moderately inclined ( $10^{\circ}$ or more) and that a smaller fraction may be highly inclined (Solanki 1987).

For an intense magnetic element associated with a flux tube containing a siphon flow, there will be either an upflow or a downflow inside the magnetic element, depending on whether it is associated with the upstream or the downstream footpoint of the flux tube. This flow will be persistent and thus should be observable, in contrast to the downflows associated with the convective collapse mechanism, which are only transient events and thus would be difficult to observe. Early observations of photospheric flux tubes were interpreted as indicating downflows within many flux tubes, but more recent observations of higher resolution are interpreted as indicating that downflows (or upflows) inside photospheric flux tubes are very weak or absent (see the reviews by Solanki 1987 and Stenflo 1989). Even the best observations to date fail to resolve individual flux tubes, however, so the evidence that there is no systematic flow inside a photospheric flux tube is not conclusive, and in any case may not apply to all photospheric flux tubes. Model calculations of the effect of siphon flows on observed line profiles are needed to help settle this issue.

The siphon flow mechanism at least has the advantage of solving one well-known problem associated with the early reports of downflows. Because all of the mass flux associated with the downflow is provided by an equal mass flux associated with the upflow at the other photospheric footpoint of the arch, there is no need to expect that the mass would have to be taken from the corona, in which case the corona would be totally drained in a matter of minutes by the many photospheric flux tubes.

This work was supported in part by the National Aeronautics and Space Administration under grant NSG-7562.

\section{References}

Hasan, S. S. 1985, Astron. Astrophys., 143, 39.

Meyer, F., and Schmidt, H. U. 1968, Zeits. Angew. Math. Mech., 48, 218.

Montesinos, B., and Thomas, J. H. 1989, Astrophys J., 337, 977.

Nordlund, A. 1986, in Small Scale Magnetic Flux Concentrations in the Solar

Photosphere, ed. W. Deinzer, M. Knölker, and H. H. Voigt (Göttingen: Vanderhoeck \& Ruprecht), pp. 83-102. 
Parker, E. N. 1975, Astrophys. J., 201, 494.

Parker, E. N. 1978, Astrophys. J., 221, 368.

Priest, E. R. 1981, in Solar Active Regions, ed. F. Q. Orrall (Boulder, CO: Colorado Associated University Press), p. 213.

Solanki, S. K. 1987, in Proc. Tenth European Regional Astronomy Meeting of the IAU. Vol. 1: The Sun, ed. L. Hejna and M. Sobotka, Publ. Astron. Inst. Czechoslovakia Acad. Sci., p. 95.

Spruit, H. C. 1979, Solar Phys., 61, 363.

Spruit, H. C., and Zweibel, E. G. 1979, Solar Phys., $62,15$.

Stenflo, J. O. 1989, Astron. Astrophys. Rev. 1, 3.

Thomas, J. H. 1984, in Small-Scale Dynamical Processes in Quiet Stellar Atmospheres, ed. S. L. Keil (Sunspot, NM: National Solar Observatory), p. 276.

Thomas, J. H. 1988, Astrophys. J., 333, 407.

Thomas, J. H. 1989, in The Physics of Magnetic Flux Ropes, ed. C. T. Russell, E. R. Priest, and C. Lee (American Geophysical Union), in press.

Thomas, J. H., and Montesinos, B. 1989, preprint.

Venkatakrishnan, P. 1985, J. Astrophys. Astron., 6, 21.

Webb, A. R., and Roberts, B. 1978, Solar Phys., 59, 249. 